\title{
Neurological Complications in Active Left-Sided Infective Endocarditis Requiring Early Surgery
}

\author{
Yolanda Carrascal ${ }^{1,2 * t}$, Bárbara Segura ${ }^{1}$, Eduardo Velasco ${ }^{1}$ and Ángel L. Guerrero ${ }^{2,3+}$ \\ ${ }^{1}$ Cardiac Surgery Department, University Hospital of Valladolid, Valladolid, Spain, ${ }^{2}$ Department of Surgery and Medicine, \\ University of Valladolid, Valladolid, Spain, ${ }^{3}$ Neurology Department, University Hospital of Valladolid, Valladolid, Spain
}

\section{OPEN ACCESS}

Edited by:

Giovanni Mariscalco,

University Hospitals of Leicester NHS

Trust, United Kingdom

Reviewed by:

Antonino S. Rubino,

University of Campania Luigi

Vanvitelli, Italy

labal Haider Jaffer,

McMaster University, Canada

${ }^{*}$ Correspondence:

Yolanda Carrascal

ycarrascal@hotmail.com

tThese authors have contributed equally to this work and share first

authorship

Specialty section:

This article was submitted to

Heart Surgery,

a section of the journal

Frontiers in Cardiovascular Medicine

Received: 28 May 2021 Accepted: 04 November 2021

Published: 03 December 2021

Citation:

Carrascal Y, Segura B, Velasco E and

Guerrero ÁL (2021) Neurological

Complications in Active Left-Sided

Infective Endocarditis Requiring Early

Surgery.

Front. Cardiovasc. Med. 8:716233.

doi: $10.3389 / f C V m .2021 .716233$
Introduction: To determine whether preoperative symptomatic neurological complication (SNC) predicts a worse prognosis of patients with active left-sided infective endocarditis who required early surgery.

Methods: We conducted a retrospective chart review and analyzed risk factors for SNCs and immediate, medium-term, and long-term mortality in patients with active left-sided infective endocarditis who required early surgery (median follow-up: 70.5 months).

Results: Of 212 included patients, preoperative SNCs occurred in $22.1 \%$. Independent risk factors for preoperative SNC included early hospital admission ( $<10$ days after symptoms onset), duration of antibiotic therapy $<7$ days, vegetation diameter $>30 \mathrm{~mm}$, preoperative chronic therapy with steroids, and peripheral embolism. A new postoperative SNC occurred in $12.7 \%$ of patients. No significant differences related to preoperative or postoperative SNCs were observed in postoperative mortality (29.8\% vs. $31.5 \%)$ or during follow-up. No significant differences in postoperative mortality were observed between hemorrhagic or ischemic SNCs. There was a nonsignificant trend to increased mortality in patients who underwent surgery within 7 days of presenting with SNC (55.5\%) compared to those who underwent surgery more than 7 days after SNC (33.3\%) $(P=0.171)$. Concomitant risk of mortality or postoperative hemorrhagic transformation increased when surgery is required during the first week after preoperative SNC $(77.5 \%$ vs. $25 \%)(P=0.017)$.

Conclusions: Patients with active left-sided infective endocarditis who need early hospital admission are at a higher risk of SNC. Mortality is higher in patients who underwent surgery within 7 days of SNC, but mortality of early surgery is acceptable after the first week of preoperative ischemic or hemorrhagic complication. We have not been able to demonstrate that preoperative nor postoperative SNCs predicted a reduced immediate, medium-term, or long-term survival in the population analyzed in this study. 


\section{INTRODUCTION}

The incidence of infective endocarditis (IE) has increased from 2.7 to 3.4 per 100,000 patients/year in Spain during the last decade (1). IE mortality, which is around $20 \%$, has not changed in recent 15 years but is higher in patients who require early surgical treatment $(2,3)$ and also when associated with a preoperative symptomatic neurological complication (SNC) such as stroke (4-7). The global incidence of cerebral embolism secondary to IE ranges between 20 and 50\%, although the Spanish National Health System reported only 9\% between 2003 and 2014 (1). Only one recent publication mentioned neurological complications secondary to IE in Spain: around $50 \%$ of patients with IE underwent surgery but this percentage dropped to $32 \%$ when preoperative clinical symptoms and imaging evidence of SNC appeared (6). This study aimed to 1 . assesses the risk factors of preoperative SNC and 2. determine whether preoperative SNC predicts a worse prognosis of patients with active leftsided infective endocarditis (ALSIE) who require early surgery. Secondary aims included the assessment of other risk factors of mortality in this sample, such as time to surgery. We evaluated the incidence and risk factors for pre- and postoperative SNC, the morbimortality associated with surgical procedures, and the medium- and long-term survival in patients with ALSIE requiring early surgical treatment during the in-hospital stay.

\section{MATERIALS AND METHODS}

This is an analytic observational study with a retrospective cohort design. Participants were included if they were diagnosed with ALSIE and required early surgical treatment. Patients with leftsided valve cured IE, right-sided IE, or cardiac device-related IE were excluded from the study (Figure 1). The study was done according to the strengthening of the reporting of observational studies in epidemiology guidelines (8). The study protocol (identification number PI19-1236) received full approval from both the local Institutional Research Review Committee and the Clinical Research Ethics Committee. Informed consent was obtained from each patient. The study period included January 2000 to May 2020. Prospectively collected demographic data and qualitative and quantitative [hemoglobin $(\mathrm{g} / \mathrm{dl})$, hematocrit $(\%)$, platelet count $(\times 103 / \mu \mathrm{l})$, white blood cell count $(\times 103 / \mu \mathrm{l})$, creatinine $(\mathrm{mg} / \mathrm{dl})$, glomerular filtration rate $\left(\mathrm{ml} / \mathrm{min} / 1.73 \mathrm{~m}^{2}\right)$, procalcitonin $(\mathrm{ng} / \mathrm{ml})$, and protein $\mathrm{C}$ reactive $(\mathrm{mg} / \mathrm{l})]$ risk factors of patients with ALSIE, who required early surgery in a tertiary academic hospital, were analyzed. These patients were classified into two groups according to the presence of preoperative SNC. The asymptomatic neurological damage related to IE (showed in systematic neuroimaging procedures in patients with IE but not corresponding with neurological clinical symptoms during exploration) does not seem to increase the risk of mortality, and it is not a clear contraindication for early surgery in patients with ALSIE (4). Therefore, we only analyzed patients with preoperative SNC. The microbiological diagnosis was determined from blood cultures and/or heart valve tissue cultures. We conducted a follow-up of surviving patients. Data were prospectively collected during the follow-up. Patients were not involved in clinical research.

\section{Definitions of the Variables Analyzed Early Surgery}

Surgery performed before completing antibiotic therapy. Following the criteria established in the guidelines of the European Society of Cardiology (9), early surgery was performed in patients with heart failure, in patients with uncontrolled local or systemic IE, and to prevent systemic embolic events.

Operative mortality was defined as death occurring within the first 30 days of surgery or during the hospital stay if the patient was not discharged before this time.

\section{Emergency Surgery}

Surgery performed within $24 \mathrm{~h}$ after the need for early surgery is determined; urgent surgery: within a few days; elective surgery: after at least 1-2 weeks of antibiotic therapy.

\section{Preoperative SNC}

Any neurological injury (classified into ischemic, hemorrhagic, brain abscess, and meningoencephalitis [associated with cerebrospinal fluid culture] according to the predominant lesion) that resulted in clinical symptoms of at least $30 \mathrm{~min}$ and was accompanied by a brain lesion detected by at least one neuroimaging study ( $\mathrm{CT}$ and/or MRI). According to the findings of the neuroimaging studies, the neurological embolic injury was categorized as macroembolic (when focal neurologic damage affected the major cerebral vessel), microembolic, or mixed.

\section{Healthcare-Associated IE}

When fever appeared $>48 \mathrm{~h}$ after hospital admission or after an invasive procedure performed within the previous 6 months.

Early-onset prosthetic valve endocarditis was a case that occurred within 1 year of valve replacement.

Time to surgery was the number of days that elapsed from the onset of clinical IE symptoms to surgery. Time to admission was the number of days between the onset of IE symptoms and hospital admission. Duration of antibiotic treatment was the number of days of adequate antimicrobial therapy. Time to neurological event was the number of days between the onset of IE symptoms and detected SNC.

\section{Prosthetic Valve Dysfunction}

Paravalvular regurgitation identified by echocardiography, with or without rocking motion of the prosthesis and/or increased transprosthetic gradient due to IE (9).

Acute kidney injury was defined according to the risk, injury, failure, loss, and end-stage criteria (10).

The surgical risk was preoperatively estimated according to the European System for Cardiac Operative Risk scales (11). 


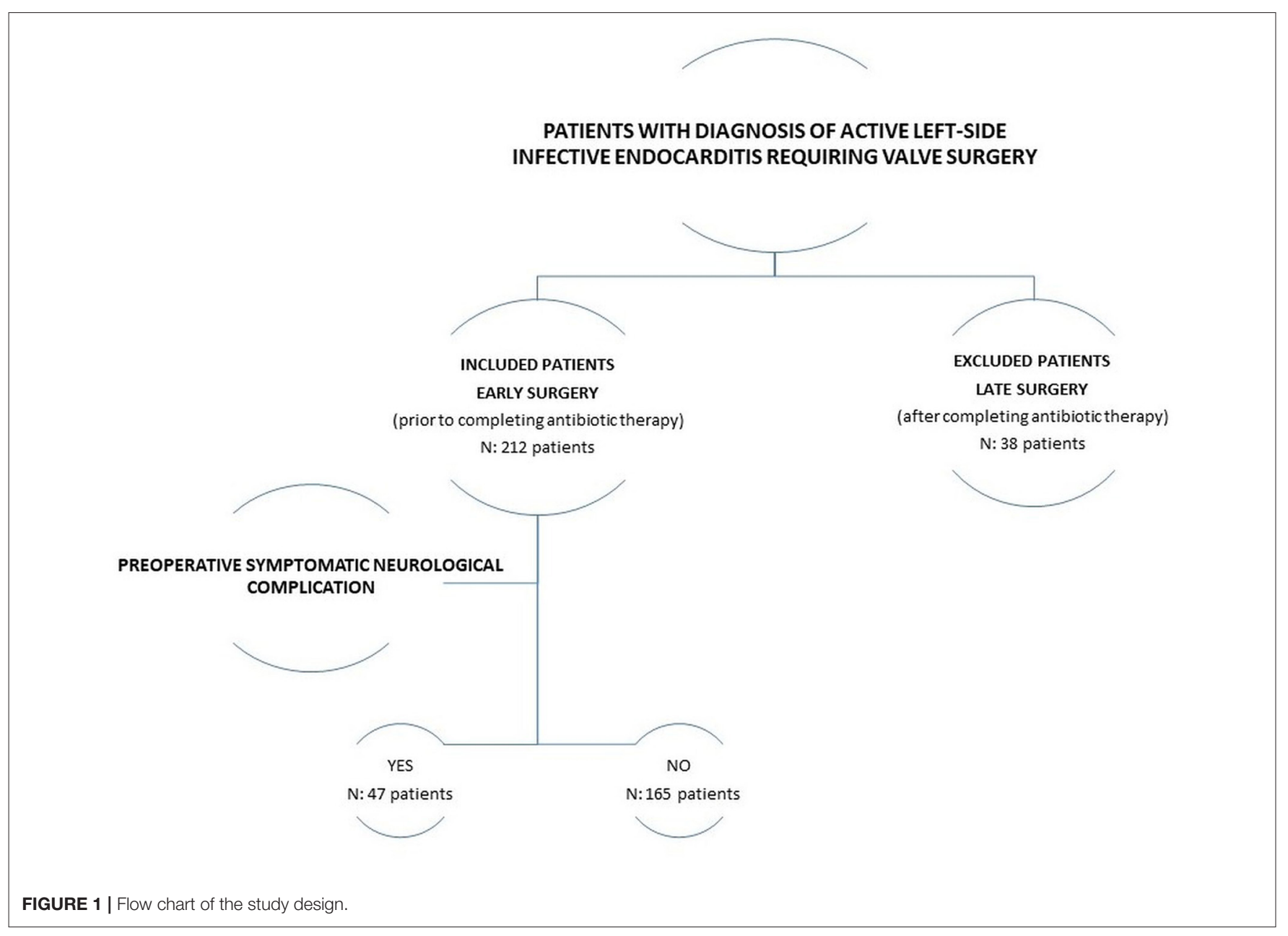

Postoperative low cardiac output was diagnosed when cardiac index was $<2.21 / \mathrm{min} / \mathrm{m}^{2}$, diuresis was $<0.5 \mathrm{ml} / \mathrm{kg} / \mathrm{h}$ and ventral venous oxygen saturation was $<60 \%$, and/or lactate was $>3$ $\mathrm{mmol} / \mathrm{l}$, in the absence of relative hypovolemia (12).

Cardiac arrhythmias were identified by electrocardiographic patterns compatible with fibrillation and atrial flutter and second- or third-degree atrial-ventricular blockages.

Postoperative SNC was diagnosed by evidence of a symptomatic embolic, thrombotic or hemorrhagic event of at least $30 \mathrm{~min}$, with or without residual motor, sensory, or cognitive deficit. Stroke was diagnosed when symptoms persisted beyond $24 \mathrm{~h}$; the transient ischemic attack was otherwise diagnosed.

The following were evaluated during follow-up: incidence of reinfection (new episode of IE caused by a different microorganism or by the same but $>6$ months after the initial episode), relapse (new episode of IE caused by the same microorganism $<6$ months after the initial episode), new SNC and redo-surgery related to IE.

\section{Statistical Analyses}

Statistical analyses were performed using SPSS software version 22.0. Quantitative variables are expressed as mean (SD), median (interquartile range) for asymmetric distributions, or as hazard ratios (95\% CI) when appropriate. The KolmogorovSmirnov test was used to determine the normality of the distribution for each variable. Qualitative variables are expressed as counts (percentages). In univariate analyses, hypothesis testing was done using the chi-squared or Fisher's exact tests for qualitative variables (the Bonferroni correction was applied for multiple comparisons) and the Student's $t$-test or Mann-Whitney $\mathrm{U}$ tests for quantitative variables. Associations between risk factors, mortality, and preoperative or postoperative neurological complications found to be significant in univariate analyses $(P$ $<0.2$ ) were entered stepwise into Cox proportional hazards models. We analyzed mortality risk factors and actuarial eventfree survival using a Cox model and the Kaplan-Meier test. A $P$ $<0.05$ was considered significant. To adjust the Cox models, we considered all preoperative variables found significant $(P<0.2)$ in the univariate analyses.

\section{RESULTS}

Demographics and Clinical Characteristics

We included 212 consecutive patients with ALSIE who required early surgery. Of these, $53.3 \%$ of patients required emergency 
TABLE 1 | Demographic profile and preoperative clinical characteristics in patients with active left-sided infective endocarditis, with or without preoperative SNC.

\begin{tabular}{lccc}
\hline Variable & No SNC & SNC & $P$ \\
$(n=165)$ & $(n=47)$ & \\
& &
\end{tabular}

\section{CLINICAL DATA}

Age (years)

Female sex

Peripheral vascular disease

Other than neurological embolism

Time to admission $<10$ days

Healthcare-associated IE

Arterial hypertension

Diabetes

Dyslipidemia

Renal failure

Haemolytic anaemia

Coronary disease

Time to SNC (days)

SNC-surgery (days)

Onset of fever-surgery (days)

Time to admission (days)

Time to surgery (days)

Haemoglobin $(\mathrm{g} / \mathrm{dL})$

\section{LOCALIZATION}

PVE

Aortic IE

Mitral IE

Multivalve IE

Early-onset PVE

MICROBIOLOGICAL DIAGNOSIS

Blood culture-negative IE

S. aureus

Methicillin-resistant $S$. aureus

S. bovis

S. agalactiae

Other streptococci

Coagulase negative staphylococci

Gram negative bacteria

HACEK species

Non-HACEK species

Enterococci

Fungi

Aerococcus

Valve culture negative

\section{ECHOCARDIOGRAPHIC FEATURES}

\begin{tabular}{lccc} 
Vegetations & $144(87.3)$ & $42(89.4)$ & 0.700 \\
Diameter < 10 mm & $38(23)$ & $7(14.9)$ & 0.229 \\
$>20 \mathrm{~mm}$ & $56(33.9)$ & $19(40.4)$ & 0.412 \\
$>30 \mathrm{~mm}$ & $10(6.1)$ & $8(17)$ & $\mathbf{0 . 0 1 7}$ \\
Annular abscess/pseudoaneurysm & $66(40)$ & $18(38.3)$ & 0.833 \\
Prosthetic valve dysfunction & $36(21.8)$ & $16(34)$ & 0.086 \\
LVEF $>50 \%$ & $140(84.8)$ & $42(89.4)$ & 0.610 \\
\hline
\end{tabular}

(Continued)
TABLE 1 | Continued

\begin{tabular}{lccc}
\hline Variable & $\begin{array}{c}\text { No SNC } \\
(\boldsymbol{n}=\mathbf{1 6 5})\end{array}$ & $\begin{array}{c}\text { SNC } \\
(\boldsymbol{n}=\mathbf{4 7})\end{array}$ & $\mathbf{P}$ \\
\hline NYHA FUNCTIONAL CLASS & & & \\
I & $8(4.8)$ & $1(2.1)$ & \\
II & $26(15.8)$ & $11(23.4)$ & 0.568 \\
II & $61(37.0)$ & $17(36.2)$ & \\
PREOPERATIVE TREATMENT & $70(42.4)$ & $18(38.3)$ & \\
Antibiotic treatment before surgery (days) & $11(14)$ & $16(11)$ & $\mathbf{0 . 0 2 3}$ \\
Antibiotic (>7 days) before surgery/SNC & $109(66.1)$ & $10(21.3)$ & $<\mathbf{0 . 0 0 0 1}$ \\
Intravenous inotropes & $53(32.1)$ & $13(27.7)$ & 0.560 \\
Anticoagulant & $40(24.2)$ & $18(38.3)$ & 0.057 \\
Calcium channel blockers & $16(9.7)$ & $1(2.1)$ & 0.128 \\
Chronic steroid treatment & $21(12.7)$ & $11(23.4)$ & 0.071 \\
INDICATIONS FOR EARLY SURGERY & & & \\
Heart failure & $119(72.1)$ & $24(51.1)$ & \\
Uncontrolled infection & $39(23.6)$ & $7(14.9)$ & $<\mathbf{0 . 0 0 0 1}$ \\
Prevention of embolism & $7(4.2)$ & $16(34)$ & \\
SCORE RISK & & & \\
Logistic EuroSCORE & $27.9(21.3)$ & $41.9(26.5)$ & $<0.0001$ \\
EuroSCORE II & $16(16.3)$ & $21.6(17.3)$ & 0.041 \\
\hline & & & \\
\hline
\end{tabular}

Qualitative values are presented as number (\%), quantitative values are presented as mean (standard deviation), or median (interquartile range) in asymmetric distributions. SNC, symptomatic neurological complication; PVE, prosthetic valve endocarditis; HACEK, Haemophilus Aggregatibacter Cardiobacterium Eikenella Kingella; LVEF, left ventricular ejection fraction. Bold values statistically significant in univariate analysis $(p<0.05)$.

surgery, the indication being heart failure in $67.5 \%$ of the cases. The preoperative characteristics are detailed in Table $\mathbf{1}$. The primary source of the infection was unknown in $51.9 \%$ of the patients, and blood cultures were sterile in $18.4 \%$. Healthcare-associated (20.3\%), gastrointestinal (10.4\%), and genitourinary $(6.2 \%)$ sources were the most common sources of primary infection. Forty-seven patients $(22.1 \%)$ presented with preoperative SNC secondary to IE (Table 2). SNC was the first clinical manifestation of IE in 21 cases (9.9\%). No fever or other typical symptoms of IE were referred by the patients before neurological event. The most frequent clinical presentation of SNC was stroke. Operative mortality was 31.1\% for all 212 patients (29.8\% in patients with preoperative SNC) $(P=0.821)$, and uncontrolled sepsis was the most frequent cause (23 cases). We conducted an in-person follow-up of all 146 surviving patients, with a mean follow-up time of $83.42 \pm 64.13$ months (median: 70.5 months, range: 1-245 months).

\section{Effect of Preoperative SNC on In-hospital Mortality}

Neurological symptoms were the first clinical manifestation of IE in $44.6 \%$ of patients with preoperative SNC diagnosis, and $<25 \%$ of those patients were receiving antibiotic therapy at that point. Only eight patients received active antibiotic treatment in the 3 days before the neurological event, and $83 \%$ of the patients received no antibiotic treatment before SNC. The risk factors related to the presence of preoperative SNC are shown 
TABLE 2 | Characteristics of patients with preoperative SNC $(n=47)$.

\begin{tabular}{lc}
\hline Variable & No. (\%) \\
\hline Ischemic stroke & $33(70.2)$ \\
Hemorrhagic stroke & $12(25.5)$ \\
Meningoencephalitis & $2(4.3)$ \\
Cerebral Imaging techniques (CT/MRI) & \\
Multiple lesions & $23(48.9)$ \\
Macroembolism & $32(68.1)$ \\
Microembolism & $8(17.0)$ \\
Both & $7(14.9)$ \\
Clinical presentation & \\
Stroke & $41(87.2)$ \\
Transitory ischemic accident & $5(10.6)$ \\
Seizures & $1(2.1)$ \\
\hline
\end{tabular}

SNC, symptomatic neurological complication; CT, computed tomography; MRI, magnetic resonance imaging.

TABLE 3 | Risk factors for preoperative SNC.

\begin{tabular}{lccc}
\hline Risk factor & HR & $\mathbf{9 5 \%} \mathbf{C l}$ & $\boldsymbol{p}$ \\
\hline Other than neurological embolism & 8.1 & $3.4-19$ & $<0.0001$ \\
Antibiotic treatment before SNC $<7$ days & 9.1 & $3.3-23.9$ & $<0.0001$ \\
Vegetation diameter $>30 \mathrm{~mm}$ & 6.4 & $1.7-24.4$ & 0.006 \\
Time to admission $<10$ days & 2.5 & $1.1-6$ & 0.029 \\
Preoperative chronic steroid treatment & 3.7 & $1.2-10.7$ & 0.015 \\
Peripheral vascular disease & 4.2 & $0.8-21.6$ & 0.078 \\
Female sex & 2.3 & $0.8-6.3$ & 0.081 \\
Preoperative anticoagulant treatment & 2.2 & $0.8-5.5$ & 0.089 \\
\hline
\end{tabular}

$P<0.05$ is considered significant. SNC, symptomatic neurological complication; HR, hazard ratio; $\mathrm{Cl}$, confidence interval.

in Table 3. The median time between preoperative SNC and surgery was 18 days (range: 4-42) in patients with hemorrhagic lesions and 15 days (range: 1-44) in patients with preoperative ischemic injury $(P=0.433)$, and mortality was 16.6 and $36.6 \%$, respectively $(P=0.206)$. About $25 \%$ of survival patients who underwent surgery within the first week (eight patients) suffered a postoperative hemorrhagic transformation compared to $12 \%$ of those who underwent surgery after the first week (25 patients) $(P=0.372)$. There was a non-significant trend to increased mortality in nine patients who underwent surgery within 7 days of presenting with SNC (55.5\%) compared to those 38 patients who underwent surgery more than 7 days after SNC (38 patients) $(33.3 \% ; P=0.171)$, but the combined risk of mortality or postoperative hemorrhagic transformation increased when surgery was required in the first week after preoperative SNC: $77.5 \%$ vs. $25 \%(P=0.017)$.

\section{Effect of Preoperative SNC on Mortality During Follow-Up}

When preoperative SNC occurred, medium-term and long-term survivals were not significantly lower (Figure 2). No differences

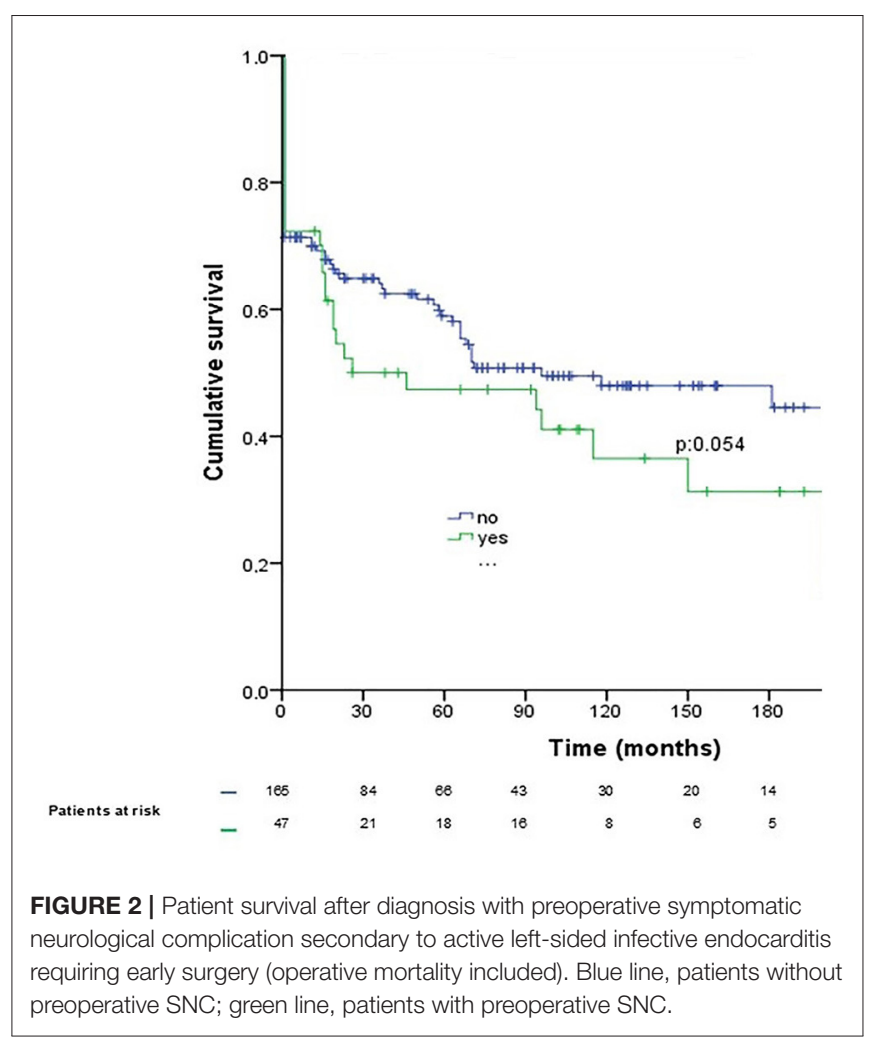

were observed between patients with postoperative SNC and the rest of the study sample (Figure 3 ).

\section{Additional Risk Factors for Mortality and Postoperative SNC}

The mortality risk factors identified in the multivariate analysis are detailed in Table 4. A new postoperative SNC was observed in only $27(12.7 \%)$ patients: $14(8.4 \%)$ in the no SNC group and $13(27.7 \%)$ in the SNC group $(P=0.001)$. Table 5 shows the risk factors for new postoperative SNC.

\section{Predictors of Mortality and SNC During Follow-Up}

A total of 45 of 146 surviving patients (30.8\%), died during follow-up period $(28.8 \%$ due to sepsis, $22.2 \%$ due to cardiac failure, $17.7 \%$ due to SNC, $15.5 \%$ due to pneumonia, $6.6 \%$ due to cancer, and $8.8 \%$ due to other causes). About $57.7 \%$ required hospital readmission with sepsis $(21.1 \%)$ being the most frequent cause. Survival at $1,5,10,15$, and 20 years was 70.5, 56.1, 45.0, 43.4, and 33.2\%, respectively. Actuarial survival of patients with ALSIE requiring early surgery is shown in Figure 2. The following were identified as risk factors for mortality during follow-up using Cox regression multivariate analysis (presented as hazard ratios [95\% CIs]): age (1.07 [1.0391.10]; $P=0.0001)$, fungal IE (8.13 [1.47-44.79]; $P=0.016)$, diameter of IE vegetation $>30 \mathrm{~mm}$ (5.17 [2.21-12.08]; $P=$ $0.0001)$, preoperative anticoagulant therapy $(2.26[1.16-4.40] ; P$ $=0.016)$, and new episode of IE (3.64 [1.47-8.96]; $P=0.002)$. 


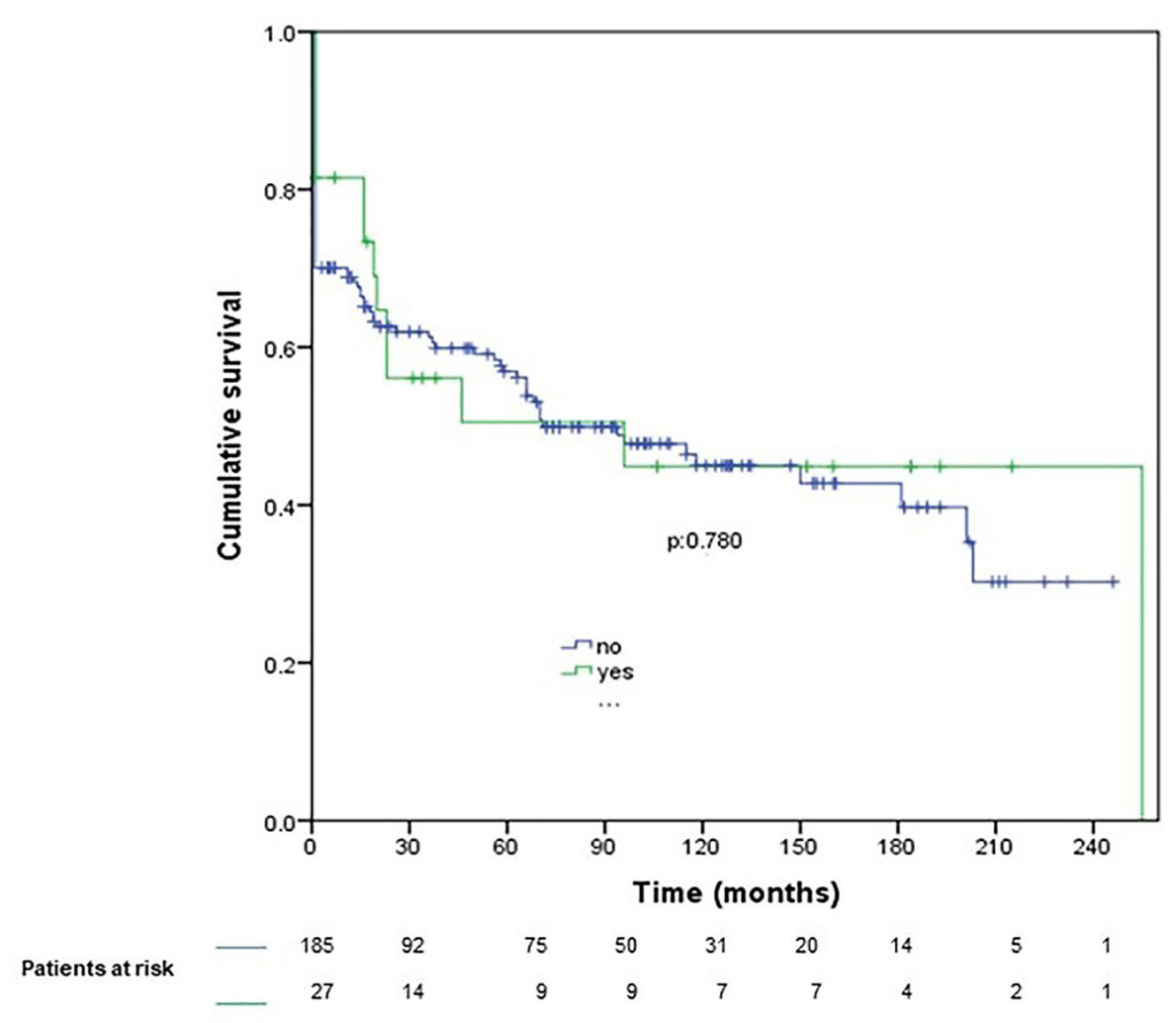

FIGURE 3 | Survival after postoperative symptomatic neurological complication (operative mortality included). Blue line, patients without postoperative SNC; green line, patients with postoperative SNC.

TABLE 4 | Risk factors for mortality.

\begin{tabular}{llll}
\hline Risk factor & HR & $\mathbf{9 5 \%} \mathbf{C l}$ & $\boldsymbol{p}$ \\
\hline Blood culture-negative IE & 2.7 & $1.1-6.7$ & 0.022 \\
Annular valve abscess & 2.6 & $1.3-5.4$ & 0.007 \\
Preoperative acute kidney injury & 4.1 & $1.6-10.1$ & 0.002 \\
Methicillin resistant infection & 7.5 & $2.1-26.7$ & 0.002 \\
Fungal endocarditis & 7.1 & $0.9-52.8$ & 0.055 \\
Age $>68$ years & 2.1 & $1.04-4.3$ & 0.037 \\
Peripheral vascular disease & 7.1 & $1.6-31.7$ & 0.010 \\
Antibiotic treatment $>7$ days & 0.2 & $0.1-0.6$ & 0.001 \\
Preoperative hemoglobin $>9.6 \mathrm{~g} / \mathrm{dll}$ & 0.3 & $0.1-0.7$ & 0.007 \\
\hline
\end{tabular}

$P<0.05$ is considered significant. HR, hazard ratio; Cl, confidence interval; IE, infective endocarditis.

Thirteen patients presented with a new IE episode: in $61.5 \%$ of cases, it was a recurrence, and in $76.9 \%$ of cases, it was due to early prosthetic valve IE. The mortality in patients diagnosed with a new IE episode was $69.2 \%$, and in $46.2 \%$ of the cases, newonset SNC was identified. Six of the patients re-admitted to the hospital with a new IE episode had preoperative SNC during the first IE episode, and five of them presented with ischemic lesions.
TABLE 5 | Risk factors for postoperative SNC in all patients and in patients without preoperative SNC.

\begin{tabular}{llll}
\hline Risk factor & HR & $\mathbf{9 5 \%} \mathbf{~ C l}$ & $\boldsymbol{p}$ \\
\hline All patients $(\boldsymbol{n}=\mathbf{2 1 2})$ & & & \\
Preoperative SNC & 2.8 & $1.0-7.2$ & 0.033 \\
Staphylococcus aureus IE & 2.8 & $1.1-7$ & 0.020 \\
Acute renal injury & 3.01 & $1.1-8.2$ & 0.031 \\
Systemic embolism & 2.5 & $1-6.6$ & 0.050 \\
Without preoperative SNC $(\boldsymbol{n}=\mathbf{1 6 5})$ & & & \\
Coronary disease & 7.02 & $1.5-32.3$ & 0.013 \\
Calcium channel blockers & 6.2 & $1.5-24.3$ & 0.008 \\
Valve annular abscess & 3.1 & $0.9-10.8$ & 0.067 \\
Other than neurological embolism & 3.06 & $0.8-10.4$ & 0.074 \\
\hline P & &
\end{tabular}

$P<0.05$ is considered significant. IE, infective endocarditis; SNC, symptomatic neurological complication; $\mathrm{HR}$, hazard ratio; $\mathrm{Cl}$, confidence interval.

The recurrence of IE (5.0 [1.1-21.5]; $P=0.031)$, Staphylococcus aureus IE (5.1 [1.3-18.9]; $P=0.014)$, early prosthetic valve IE (4.4 [1.0-19.5]; $P=0.048$ ), diabetes mellitus (5.3 [1.3-21.4]; $P=0.019$ ), and chronic steroid treatment (3.5 [1.0-12.7]; $P=$ 0.049 ) are risk factors for new SNC during follow-up (presented as hazard ratios [95\% CIs]). 


\section{DISCUSSION}

The risk of postoperative SNC increased in patients with preoperative SNC, but we have not been able to demonstrate that preoperative nor postoperative SNC predicted a reduced immediate, medium-term, or long-term survival in the analyzed population. Mortality is higher in patients who underwent surgery within 7 days of SNC, but mortality of early surgery is acceptable after the first week of preoperative ischemic or hemorrhagic complication. Mortality found in this study in patients with ALSIE is consistent with previous reports (1-6). Lower mortalities are reported in series that exclude patients with preoperative cardiogenic shock, hemorrhagic neurological complication, or without antibiotic treatment at the time of surgery (13). In the analyzed sample, patient survival depended on the pathogen virulence (14) and factors inherent to the patient: age, associated comorbidities, and preoperative clinical status. Operative mortality almost tripled among patients with negative blood cultures, while treatment with bactericidal antibiotics for at least 7 days before surgery reduced mortality by four times.

No significant differences in mortality attributable to SNC were found, despite that these patients more frequently required early surgery (i.e., before completing seven days of antibiotic treatment). For patients with IE, consensus guidelines recommend delaying surgery until 2 weeks after an ischemic event and 4 weeks after hemorrhagic damage $(9,15)$, but some researchers have not observed the benefit in delaying surgery beyond 2 weeks to prevent hemorrhagic transformation or postoperative neurological deterioration after intracranial hemorrhage $(16,17)$. In the analyzed sample, the prevalence of hemorrhagic damage is higher than in other reports $(5,6$, $18,19)$, but we did not observe higher mortality in patients with hemorrhagic damage than in patients with ischemic injury. However, we did observe an increased combined risk of mortality and/or hemorrhagic transformation when surgery was required within the first week of diagnosis with SNC. This would suggest that every effort should be made to delay surgery in patients with ALSIE within the first week of diagnosis with SNC.

Neurological symptoms were the first clinical manifestation of IE in almost half of the patients with preoperative SNC diagnosis, and less than a quarter were receiving antibiotic therapy at that point. We have identified a group of patients with ALSIE with a particularly aggressive disease progression: those requiring hospitalization within 10 days of IE symptom onset, in whom the probability of SNC is 2.5 times greater. Heart failure is the most frequent cause of early surgery in this group, as well as in patients with a more favorable disease course $(70 \%$ vs. $64.7 \%$, respectively; $P=0.67$ ), but the median number of days from the onset of symptoms to surgery was significantly lower (18 vs. 43; $P<0.0001)$.

The risk factors for postoperative SNC in our sample were generally related to previous embolic episodes, a preoperative symptomatic low cardiac output, and a worse prognosis of
IE measured by a rapid disease progression as seen in other studies (4). However, the analysis of medium- and longterm survival of patients with preoperative and postoperative SNC did not show significantly higher mortality than those without SNC. However, we cannot absolutely exclude that the prognosis is worse in a larger sample of patients. During follow-up, IE recurrence was the main risk factor for mortality and new SNC in patients with ALSIE who had undergone early surgery.

\section{Limitations}

These results might not be extrapolated to larger populations with different demographic and clinical characteristics. Selection bias is possible due to the low frequency of surgery in patients with SNC secondary to IE in Spain (6). Besides, the reason for severe complications that required surgical treatment would appear at different time intervals for each patient, regardless of whether they have a previous SNC and how long it has evolved. There is no doubt that all the patients included in the study meet the inclusion criteria for early surgery, nevertheless, possible bias secondary to the elapsed time between SNC and severe complications of IE who need early surgery is impossible to control. High mortality during surgery, due to preoperative cardiogenic shock, makes it difficult to tease out the effects of preoperative SNC. Finally, this is an observational study, so no cause-effect relationships can be determined at this point.

\section{CONCLUSIONS}

Patients with ALSIE who need early hospital admission rapidly progress toward SNC. The combined risk of mortality or postoperative hemorrhagic transformation is higher in patients who underwent surgery within 7 days of SNC, but mortality of early surgery is acceptable after the first week of preoperative ischemic or hemorrhagic SNC. We have not been able to demonstrate that preoperative SNCs predicted a reduced immediate, medium-term, or long-term survival in the population analyzed in this study. No differences were identified in immediate, medium-term, or long-term survival after postoperative SNC. ALSIE recurrence is the main risk factor that jointly contributes to mortality and new SNC during follow-up.

\section{DATA AVAILABILITY STATEMENT}

The original contributions presented in the study are included in the article/supplementary material, further inquiries can be directed to the corresponding author/s.

\section{ETHICS STATEMENT}

The study protocol (identification number PI19-1236) received full approval by both the local Institutional Research Review Committee and the Clinical Research Ethics Committee of Hospital Clínico Universitario de Valladolid. Avda. Ramón y Cajal 3. 47003 Valladolid Spain. The patients/participants 
provided their written informed consent to participate in this study.

\section{AUTHOR CONTRIBUTIONS}

YC and ÁG have contributed to the design, planning, conduct, and reporting of the work described in the article. BS and EV

\section{REFERENCES}

1. Olmos C, Vilacosta I, Fernández-Pérez C, Bernal JL, Ferrera C, GarcíaArribas D, et al. The evolving nature of infective endocarditis in Spain: a population-based study (2003 to 2014). J Am Coll Cardiol. (2017) 22:2795804. doi: 10.1016/j.jacc.2017.10.005

2. Prendergast BD, Tornos P. Surgery for infective endocarditis: who and when? Circulation. (2010) 121:114152. doi: 10.1161/CIRCULATIONAHA.108.773598

3. Di Mauro M, Dato GMA, Barili F, Gelsomino S, Santè P, Corte AD, et al. A predictive model for early mortality after surgical treatment of heart valve or prosthesis infective endocarditis. Int J Cardiol. (2017) 241:97102. doi: 10.1016/j.ijcard.2017.03.148

4. Selton-Suty C, Delahaye F, Tattevin P, Federspiel C, Le Moing V, Chirouze C, et al. Symptomatic and asymptomatic neurological complications of infective endocarditis: impact on surgical management and prognosis. PLOS ONE. (2016) 11:e0158522. doi: 10.1371/journal.pone.0158522

5. Okita Y, Minakata K, Yasuno S, Uozumi R, Sato T Ueshima K, et al. Optimal timing of surgery for active infective endocarditis with cerebral complications: a Japanese multicentre study. Eur J Cardiothorac Surg. (2016) 50:374-82. doi: 10.1093/ejcts/ezw035

6. García-Cabrera E, Fernández-Hidalgo N, Almirante B, IvanovaGeorgieva R, Noureddine M, Plata A, et al. Neurological complications of infective endocarditis: risk factors, outcome, and impact of cardiac surgery: a multicenter observational study. Circulation. (2013) 127:272-84. doi: 10.1161/CIRCULATIONAHA.112.000813

7. Carneiro TS, Awtry E, Dobrilovic N, Fagan MA, Kimmel S, Weinstein ZM, et al. Neurological complications of endocarditis: a multidisciplinary review with focus on surgical decision making. Semin Neurol. (2019) 39:495506. doi: $10.1055 / \mathrm{s}-0039-1688826$

8. von Elm E, Altman DG, Egger M, Pocock SJ, Gøtzsche PC, Vandenbroucke JP, et al. The Strengthening the Reporting of Observational Studies in Epidemiology (STROBE) statement: guidelines for reporting observational studies. PLoS Med. (2007) 4:e296. doi: 10.1371/journal.pmed.0040296

9. Habib G, Lancellotti P, Antunes MJ, Bongiorni MG, Casalta JP, Del Zotti F, et al. 2015 ESC guidelines for the management of infective endocarditis: the task force for the management of infective endocarditis of the European Society of Cardiology (ESC). Eur Heart J. (2015). 36: 3075128. doi: 10.1093/eurheartj/ehv319

10. Bellomo R, Ronco C, Kellum JA, Mehta RL, Palevsky P. Acute Dialysis Quality Initiative workgroup. acute renal failure - definition, outcome measures, animal models, fluid therapy and information technology needs: the Second International Consensus Conference of the Acute Dialysis Quality Initiative (ADQI) Group. Crit Care. (2004) 8:R204-12. doi: 10.1186/cc2872

11. Nashef SA, Roques F, Michel P, Gauducheau E, Lemeshow S, Salamon R. European system for cardiac operative risk evaluation (EuroSCORE). Eur J Cardiothorac Surg. (1999) 16:9-13. doi: 10.1016/S1010-7940(99) 00134-7 have contributed to the conduct and reporting of the work described in this article. All authors have finally approved the manuscript.

\section{ACKNOWLEDGMENTS}

We would like to thank Jennifer Wall for editing this article.

12. Pérez Vela JL, Martín Benítez JC, Carrasco González M, de la Cal López MA, Hinojosa Pérez R, Sagredo Meneses V, et al. Clinical practice guide for the management of low cardiac output syndrome in the postoperative period of heart surgery. Med Intensiva. (2012) 36:e144. doi: 10.1016/j.medin.2012.02.007

13. Hussain ST, Shrestha NK, Gordon SM, Houghtaling PL, Blackstone EH, Pettersson GB. Residual patient, anatomic, and surgical obstacles in treating active left-sided infective endocarditis. J Thorac Cardiovasc Surg. (2014) 148:981-8. doi: 10.1016/j.jtcvs.2014.06.019

14. Wang A, Gaca JC, Chu VH. Management considerations in infective endocarditis: a review. JAMA. (2018) 320:72-83. doi: 10.1001/jama.2018.7596

15. Petterson GB, Coselli JS. 2016 The American Association for Thoracic Surgery (AATS) consensus guidelines: surgical treatment of infective endocarditis: Executive summary. J Thorac Cardiovasc Surg. (2017) 153:124158. doi: 10.1016/j.jtcvs.2016.09.093

16. Yoshioka D, Sakaguchi T, Yamauchi T, Okazaki S, Miyagawa S, Nishi H, et al. Impact of early surgical treatment on postoperative neurologic outcome for active infective endocarditis complicated by cerebral infarction. Ann Thorac Surg. (2012) 94:489-96. doi: 10.1016/j.athoracsur.2012.04.027

17. Wilbring $M$, Irmscher L, Alexiou K, Matschke K, Tugtekin SM. The impact of preoperative neurological events in patients suffering from native infective valve endocarditis. Interact Cardiovasc Thorac Surg. (2014) 18:7407. doi: 10.1093/icvts/ivu039

18. Ting W, Silverman N, Levitsky S. Valve replacement in patients with endocarditis and cerebral septic emboli. Ann Thorac Surg. (1991) 51:1821. doi: 10.1016/0003-4975(91)90439-W

19. Derex L, Bonnefoy E, Delahaye F. Impact of stroke on therapeutic decision making in infective endocarditis. J Neurol. (2010) 257:31521. doi: $10.1007 / \mathrm{s} 00415-009-5364-3$

Conflict of Interest: The authors declare that the research was conducted in the absence of any commercial or financial relationships that could be construed as a potential conflict of interest.

Publisher's Note: All claims expressed in this article are solely those of the authors and do not necessarily represent those of their affiliated organizations, or those of the publisher, the editors and the reviewers. Any product that may be evaluated in this article, or claim that may be made by its manufacturer, is not guaranteed or endorsed by the publisher.

Copyright (c) 2021 Carrascal, Segura, Velasco and Guerrero. This is an open-access article distributed under the terms of the Creative Commons Attribution License (CC $B Y)$. The use, distribution or reproduction in other forums is permitted, provided the original author(s) and the copyright owner(s) are credited and that the original publication in this journal is cited, in accordance with accepted academic practice. No use, distribution or reproduction is permitted which does not comply with these terms. 